\title{
MCEE Mutations in an Adult Patient with Parkinson's Disease, Dementia, Stroke and Elevated Levels of Methylmalonic Acid
}

\author{
Mattias Andréasson 1,2,3,*iD, Rolf H. Zetterström ${ }^{4,5}$, Ulrika von Döbeln ${ }^{4}$, Anna Wedell ${ }^{4,5}$ \\ and Per Svenningsson 1,2,3 \\ 1 Department of Neurology, Karolinska University Hospital, 14186 Stockholm, Sweden; \\ per.svenningsson@ki.se \\ 2 Department of Clinical Neuroscience, Karolinska Institutet, 17177 Stockholm, Sweden \\ 3 Center for Neurology, Academic Specialist Center, 11365 Stockholm, Sweden \\ 4 Center for Inherited Metabolic Diseases, Karolinska University Hospital, 17176 Stockholm, Sweden; \\ rolf.zetterstrom@ki.se (R.H.Z.); ulrika.dobeln@ki.se (U.v.D.); anna.wedell@ki.se (A.W.) \\ 5 Department of Molecular Medicine and Surgery, Karolinska Institutet, 17176 Stockholm, Sweden \\ * Correspondence: mattias.andreasson@ki.se
}

Received: 8 May 2019; Accepted: 27 May 2019; Published: 29 May 2019

\begin{abstract}
Methylmalonic aciduria (MMA-uria) is seen in several inborn errors of metabolism (IEM) affecting intracellular cobalamin pathways. Methylmalonyl-CoA epimerase (MCE) is an enzyme involved in the mitochondrial cobalamin-dependent pathway generating succinyl-CoA. Homozygous mutations in the corresponding MCEE gene have been shown in children to cause MCE deficiency with isolated MMA-uria and a variable clinical phenotype. We describe a 78-year-old man with Parkinson's disease, dementia and stroke in whom elevated serum levels of methylmalonic acid had been evident for many years. Metabolic work-up revealed intermittent MMA-uria and increased plasma levels of propionyl-carnitine not responsive to treatment with high-dose hydroxycobalamin. Whole genome sequencing was performed, with data analysis targeted towards genes known to cause IEM. Compound heterozygous mutations were identified in the MCEE gene, c.139C $>\mathrm{T}$ (p.Arg47X) and c.419delA (p.Lys140fs), of which the latter is novel. To our knowledge, this is the first report of an adult patient with MCEE mutations and MMA-uria, thus adding novel data to the possible phenotypical spectrum of MCE deficiency. Although clinical implications are uncertain, it can be speculated whether intermittent hyperammonemia during episodes of metabolic stress could have precipitated the patient's ongoing neurodegeneration attributed to Parkinson's disease.
\end{abstract}

Keywords: methylmalonic acidemia; methylmalonic acid; methyl-CoA epimerase; MCEE gene; cobalamin

\section{Introduction}

Following cellular uptake into the lysosome, cobalamin is converted to adenosylcobalamin (AdoCbl) and methylcobalamin $(\mathrm{MeCbl})$ through two separate pathways. MeCbl functions as a co-factor in the cytosolic methionine cycle and AdoCbl serves as a co-factor for the mitochondrial enzyme methylmalonyl-CoA mutase (MUT) [1,2]. Methylmalonic aciduria (MMA-uria) is present in several inborn errors of metabolism (IEM) affecting different steps of these intracellular cobalamin pathways. In particular, disruption of AdoCbl, and by extension MUT, is known to cause isolated MMA-uria [2]. Stroke affecting the basal ganglia, movement disorders and cognitive decline have been described in the context of isolated MMA-uria [3]. 
Methylmalonyl-CoA epimerase (MCE) is an enzyme participating, together with MUT, in the mitochondrial pathway metabolizing propionyl-CoA to succinyl-CoA, which in turn enters the citric acid cycle. MCE acts upstream from MUT, converting D-methylmalonyl-CoA to L-methylmalonyl-CoA (Figure 1). Thus, MCE deficiency is also thought to cause isolated MMA-uria [1,4].

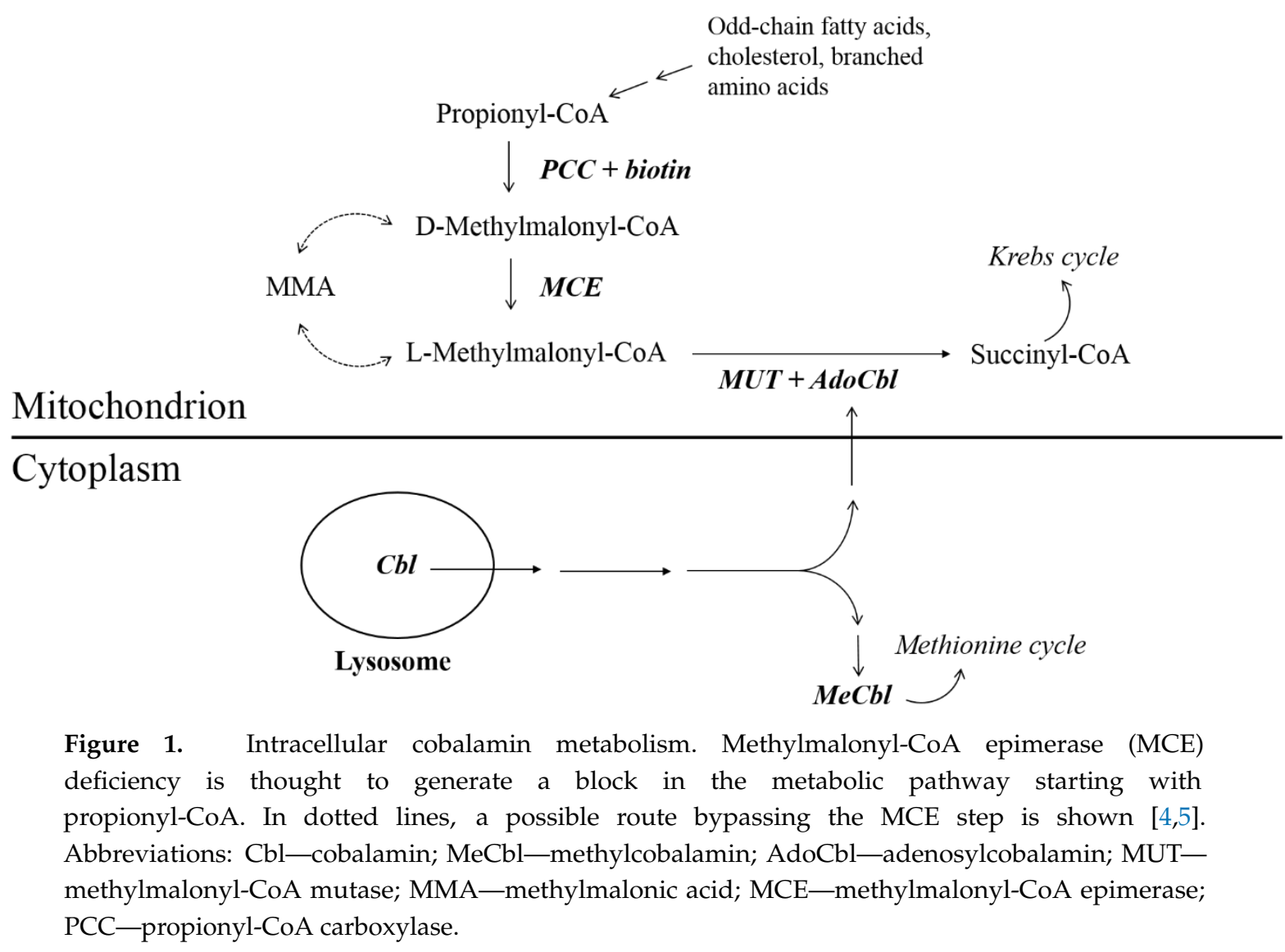

MCE deficiency, with underlying MCEE mutations, has been described in at least 18 children, with phenotypes ranging from acute metabolic acidosis to asymptomatic presentations [4,6-10]. With this case report, we describe an adult patient with MMA-uria and compound heterozygous mutations in the MCEE gene, of which one is novel, and discuss possible implications with regard to the patient's concurrent Parkinson's disease (PD).

\section{Case Report}

The patient and his two sons have signed informed consents. Patient-related procedures have been approved by the regional ethical board (reference number: 2016/19-31/2, date of approval: 21 March 2016).

The proband is a 78-year-old man of German descent. Family history consists of a mother with dementia at an unknown age. A diagnosis of PD was made in 2004 with satisfactory motor response to dopaminergic treatment. Moreover, the patient has a known medical history of chronic renal failure, cardiomyopathy, hypertension, asthma, prostatic cancer, infratentorial stroke in 2009 and a cortical stroke in 2016. Brain magnetic resonance imaging (MRI) in 2014 demonstrated older basal ganglia infarctions.

In 2007, milder short-term memory difficulties were reported and the patient exhibited a Mini Mental State Examination (MMSE) score of 28/30. Treatment was initiated with memantine but transient episodes of confusion emerged and by the end of 2011, donepezil was added. Cognitive deterioration continued with a Montreal Cognitive Assessment (MoCA) score of 17/30 in 2013. Behavioral symptoms 
ensued, including hallucinations, anxiety and delusions, causing the patient to be moved to a nursing home. At examination in 2016, the patient exhibited asymmetric parkinsonism with intermittent rest tremor, impaired postural control, a MoCA score of 5/30 and he needed a walker.

Due to markedly elevated levels of methylmalonic acid (MMA) in serum, a metabolic investigation was undertaken in 2015. First, a treatment trial with hydroxycobalamin categorized the patient's biochemical profile as a non-responder to cobalamin substitution. Mild MMA-uria, together with increased plasma levels of propionyl-carnitine, were demonstrated in 2016 (Table 1).

Table 1. Biochemical parameters reflecting status of the methionine cycle, renal function and mitochondrial metabolism with regard to methylmalonic acid (MMA). Chronic elevation of MMA is seen, together with mild MMA-uria and an associated elevation of p-propionyl-carnitine in 2016. Propionyl-carnitine was determined with a modification of the LC-MS-MS-method described by Ghoshal et al. [11], and u-methylmalonate by GC-MS-analysis of organic acids in urine [12].

\begin{tabular}{|c|c|c|c|c|c|}
\hline Biochemical Analyses & $\begin{array}{c}2005 \\
\text { Apr, Dec }\end{array}$ & $\begin{array}{l}2011 \\
\text { Mar }\end{array}$ & $\begin{array}{c}2013 \\
\text { Mar, Oct }\end{array}$ & $\begin{array}{c}2015 \\
\text { Oct, Dec }\end{array}$ & $\begin{array}{c}2016 \\
\text { Jan, Jun }\end{array}$ \\
\hline MMA $(\mu \mathrm{mol} / 1)[<0,40]$ & $8.1^{a}, 11^{a}$ & $180^{a}$ &,$- 35^{a}$ & $9.9^{b}, 16^{b, e}$ &,$- \mathbf{1 8}^{\mathrm{b}}$ \\
\hline p-Hcy $(\mu \mathrm{mol} / \mathrm{l})[5,0-15]^{\mathrm{c}},[5,0-20]^{\mathrm{d}}$ & & & $17^{\mathrm{c}}, 18^{\mathrm{c}}$ & $26^{d}, 24^{d, e}$ &,$- 32^{c}$ \\
\hline s-cobalamin (pmol/l) [150-650] & 510,460 & 420 &,- 410 & & \\
\hline s-folate $(\mathrm{nmol} / \mathrm{l})[7-40]$ & $>40,-$ & $>40$ & & & \\
\hline p-propionyl-carnitine $(\mu \mathrm{mol} / \mathrm{l})[<1,3]$ & & & & &,- 13 \\
\hline u-methylmalonate $(\mathrm{mmol} / \mathrm{mol}$ creatinine) $[<10]$ & & & & $9.0,5.5$ & 10,60 \\
\hline p-creatinine $(\mu \mathrm{mol} / \mathrm{l})[<100]$ &,- 124 & 117 & 119,133 &,- 189 & $159,-$ \\
\hline
\end{tabular}

Abbreviations: MMA-methylmalonic acid; Hcy-homocysteine. ${ }^{a}$ serum; ${ }^{b}$ plasma; ${ }^{c, d}$ different homocysteine reference intervals; ${ }^{\mathrm{e}}$ measured after treatment with high dose hydroxycobalamin.

Clinical whole genome sequencing ensued, and the subsequent data were analyzed with the Mutation Identification Pipeline (MIP, v3.0.7). The data analysis was in silico, restricted to 681 genes known to cause IEM, as previously described [13]. Two heterozygous mutations were identified in the MCEE gene and were validated by Sanger sequencing. The first mutation, c.139C $>\mathrm{T}$ (p.Arg47X), is identical to the first described homozygous mutation in MCE deficiency [6]. The second mutation, c.419delA (p.Lys140fs), causes a frameshift introducing a premature stop codon that has not been described before in the context of MCE deficiency (Figure 2). 


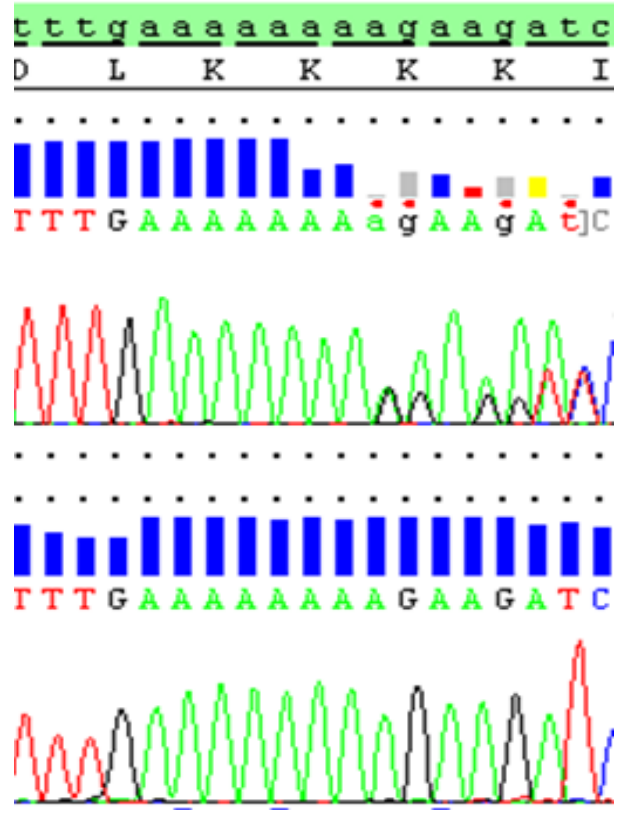

Figure 2. Chromatogram from Sanger DNA sequencing showing the novel c.419delA mutation in the $M C E E$ gene in heterozygous form, as detected in the proband (top). The mutation causes a frameshift at Lys (K) 140 leading to a premature stop codon in the protein. Below is the normal reference sequence for comparison.

No significant clinical or biochemical deviations were demonstrated in the proband's two sons. Sanger sequencing of the MCEE gene, in genomic DNA isolated from blood, revealed the sons as heterozygous carriers of one each of the proband's mutations.

\section{Discussion}

We describe an adult patient with highly elevated plasma MMA and mild MMA-uria likely caused by an underlying MCE deficiency due to compound heterozygous mutations in the MCEE gene. Cellular complementation studies and $\left[{ }^{14} \mathrm{C}\right]$ propionate incorporation assays were not performed in this patient. However, one of the mutations, c.139C $>\mathrm{T}$ (p.Arg47X), is a nonsense mutation that has been shown to cause MCE deficiency in homozygous state, and the other is a frameshift mutation causing a premature stop codon. Both mutations are thus predicted to result in the synthesis of truncated versions of the MCE protein, supporting an underlying MCE deficiency explaining the patient's biochemical phenotype $[4,6]$.

The patient demonstrated a modest hyperhomocysteinemia that gradually increased with time and paralleled the patient's deteriorating renal function. The elevated homocysteine levels persisted despite high-dose substitution with hydroxycobalamin, thereby precluding an underlying cobalamin deficiency (Table 1 ; footnote ${ }^{\mathrm{e}}$ ). Thus, the hyperhomocysteinemia was mainly considered to reflect a gradually decreasing glomerular filtration rate.

To our knowledge, this is the first clinico-genetic characterization of an adult with isolated MMA-uria with underlying MCEE mutations. We cannot conclude for certain whether the patient's presumptive MCE deficiency is clinically symptomatic. In theory, increased levels of propionyl-CoA inhibit the enzyme $\mathrm{N}$-acetylglutamate synthase, which is essential for maintaining the urea cycle. In turn, subsequent impairment of the urea cycle could contribute to hyperammonemia which is a known toxic state for the central nervous system [14]. Thus, it can be speculated whether intermittent hyperammonemia during episodes of metabolic stress, and increased loading of MCE-dependent metabolic pathways, might have contributed to or precipitated the patient's concurrent neurodegeneration attributed to PD. Unfortunately, no testing for hyperammonemia was performed in the described patient. Furthermore, repeated blood gas analysis was not carried out, except in 2014, 
in which normal pCO2 and lactate levels were seen in venous blood. However, it is notable that the patient already developed cognitive impairment within 3 years after diagnosis of $\mathrm{PD}$, in spite of normal cerebrospinal fluid markers (tau, phosphorylated tau and $\beta$-amyloid) in 2012.

Previous reports of MCE deficiency have included cases with clinically mild phenotypes, which in turn has questioned the clinical relevance of MCE deficiency [10]. Furthermore, the presence of an alternative compensatory metabolic route for D-methylmalonyl-CoA, bypassing the MCE step, has been proposed and thus possibly partly explains the existence of mild phenotypes (Figure 1) [4].

\section{Conclusions}

We report a case of MCE deficiency in an adult patient with concurrent PD, stroke and dementia exhibiting highly elevated plasma MMA and mild intermittent MMA-uria. One of the identified underlying compound heterozygous mutations in the MCEE gene is novel. Further characterization of phenotypes in adult patients with MCE deficiency are needed in order to better understand possible clinical implications with regard to concurrent neurodegenerative diseases such as PD.

Author Contributions: M.A.: planning, clinical investigation, analysis and interpretation of data, drafting of manuscript. P.S.: planning, clinical investigation, analysis and interpretation of data, manuscript revision, funding acquisition. R.H.Z.: analysis and interpretation of data, manuscript revision. U.v.D.: analysis and interpretation of data, manuscript revision. A.W.: analysis and interpretation of data, manuscript revision.

Funding: This study was funded by ALF from Stockholm City Council. Anna Wedell and Per Svenningsson are Wallenberg Clinical Scholars.

Acknowledgments: We thank the patient and his two sons for participation in this study.

Conflicts of Interest: The authors declare no conflict of interest relevant to this work. The funders had no role in the design of the study; in the collection, analyses, or interpretation of data; in the writing of the manuscript, or in the decision to publish the results.

\section{References}

1. Hannibal, L.; Lysne, V.; Bjorke-Monsen, A.L.; Behringer, S.; Grunert, S.C.; Spiekerkoetter, U.; Jacobsen, D.W.; Blom, H.J. Biomarkers and algorithms for the diagnosis of vitamin b12 deficiency. Front. Mol. Biosci. 2016, 3, 27. [CrossRef] [PubMed]

2. Watkins, D.; Rosenblatt, D.S. Inborn errors of cobalamin absorption and metabolism. Am. J. Med. Genet. C Semin. Med. Genet. 2011, 157C, 33-44. [CrossRef] [PubMed]

3. Ktena, Y.P.; Paul, S.M.; Hauser, N.S.; Sloan, J.L.; Gropman, A.; Manoli, I.; Venditti, C.P. Delineating the spectrum of impairments, disabilities, and rehabilitation needs in methylmalonic acidemia (mma). Am. J. Med. Genet. A 2015, 167A, 2075-2084. [CrossRef] [PubMed]

4. Dobson, C.M.; Gradinger, A.; Longo, N.; Wu, X.; Leclerc, D.; Lerner-Ellis, J.; Lemieux, M.; Belair, C.; Watkins, D.; Rosenblatt, D.S.; et al. Homozygous nonsense mutation in the mcee gene and sirna suppression of methylmalonyl-coa epimerase expression: A novel cause of mild methylmalonic aciduria. Mol. Genet. Metab. 2006, 88, 327-333. [CrossRef] [PubMed]

5. Montgomery, J.A.; Mamer, O.A.; Scriver, C.R. Metabolism of methylmalonic acid in rats. Is methylmalonyl-coenzyme a racemase deficiency symptomatic in man? J. Clin. Invest. 1983, 72, 1937-1947. [CrossRef] [PubMed]

6. Bikker, H.; Bakker, H.D.; Abeling, N.G.; Poll-The, B.T.; Kleijer, W.J.; Rosenblatt, D.S.; Waterham, H.R.; Wanders, R.J.; Duran, M. A homozygous nonsense mutation in the methylmalonyl-coa epimerase gene (mcee) results in mild methylmalonic aciduria. Hum. Mutat. 2006, 27, 640-643. [CrossRef] [PubMed]

7. Gradinger, A.B.; Belair, C.; Worgan, L.C.; Li, C.D.; Lavallee, J.; Roquis, D.; Watkins, D.; Rosenblatt, D.S. Atypical methylmalonic aciduria: Frequency of mutations in the methylmalonyl coa epimerase gene (mcee). Hum. Mutat. 2007, 28, 1045. [CrossRef] [PubMed]

8. Waters, P.J.; Thuriot, F.; Clarke, J.T.; Gravel, S.; Watkins, D.; Rosenblatt, D.S.; Levesque, S. Methylmalonyl-coa epimerase deficiency: A new case, with an acute metabolic presentation and an intronic splicing mutation in the mcee gene. Mol. Genet. Metab. Rep. 2016, 9, 19-24. [CrossRef] [PubMed] 
9. Abily-Donval, L.; Torre, S.; Samson, A.; Sudrié-Arnaud, B.; Acquaviva, C.; Guerrot, A.M.; Benoist, J.F.; Marret, S.; Bekri, S.; Tebani, A. Methylmalonyl-coa epimerase deficiency mimicking propionic aciduria. Int. J. Mol. Sci. 2017, 18. [CrossRef] [PubMed]

10. Heuberger, K.; Bailey, H.J.; Burda, P.; Chaikuad, A.; Krysztofinska, E.; Suormala, T.; Burer, C.; Lutz, S.; Fowler, B.; Froese, D.S.; et al. Genetic, structural, and functional analysis of pathogenic variations causing methylmalonyl-coa epimerase deficiency. Biochim. Biophys. Acta Mol. Basis Dis. 2019. [CrossRef] [PubMed]

11. Ghoshal, A.K.; Guo, T.; Soukhova, N.; Soldin, S.J. Rapid measurement of plasma acylcarnitines by liquid chromatography-tandem mass spectrometry without derivatization. Clin. Chim. Acta 2005, 358, 104-112. [CrossRef] [PubMed]

12. Alm, J.; Hagenfeldt, L.; Larsson, A. Concentrations of organic acids in the urine of healthy newborn children. Ann. Clin. Biochem. 1978, 15, 245-249. [CrossRef] [PubMed]

13. Stranneheim, H.; Engvall, M.; Naess, K.; Lesko, N.; Larsson, P.; Dahlberg, M.; Andeer, R.; Wredenberg, A.; Freyer, C.; Barbaro, M.; et al. Rapid pulsed whole genome sequencing for comprehensive acute diagnostics of inborn errors of metabolism. BMC Genomics 2014, 15, 1090. [CrossRef] [PubMed]

14. Dercksen, M.; IJlst, L.; Duran, M.; Mienie, L.J.; van Cruchten, A.; van der Westhuizen, F.H.; Wanders, R.J. Inhibition of n-acetylglutamate synthase by various monocarboxylic and dicarboxylic short-chain coenzyme a esters and the production of alternative glutamate esters. Biochim. Biophys. Acta 2014, 1842, 2510-2516. [CrossRef] [PubMed]

(C) 2019 by the authors. Licensee MDPI, Basel, Switzerland. This article is an open access article distributed under the terms and conditions of the Creative Commons Attribution (CC BY) license (http://creativecommons.org/licenses/by/4.0/). 